\title{
Research on Dynamic Structure Emergency Resource Integration Based on Information Fusion
}

\author{
Wang Guohua, Zhou Jiahui, Cui Zhe* \\ School of Business, Nantong University, Nantong, China \\ Email address: \\ 7790@vip.163.com (Cui Zhe) \\ ${ }^{*}$ Corresponding author
}

To cite this article:

Wang Guohua, Zhou Jiahui, Cui Zhe. Research on Dynamic Structure Emergency Resource Integration Based on Information Fusion. International Journal of Economics, Finance and Management Sciences. Vol. 5, No. 3, 2017, pp. 189-193. doi: 10.11648/j.ijefm.20170503.18

Received: April 7, 2017; Accepted: April 24, 2017; Published: June 12, 2017

\begin{abstract}
Emergency decision, especially emergency resources dispatching decision is one of the elementary questions in emergency management. The paper takes dynamicity of emergency resources into consideration and gives a multi-stage dispatching thought, which includes the process of two-stage emergency rescure and continuous comsumption dynamic structure, on the basis of emergency resources' identification and integration.
\end{abstract}

Keywords: Incident, Emergency Management, Emergency Decision, Resources Dispatching

\section{Introduction}

In recent years, various public emergencies become the focus of the country and the public for many times and even become the shade of China's social economy life to some extent. With the consecutive researches on emergency management, the optimal allocation of emergency resources has strategic position in more and more countries and states in order to maximize the function of emergency rescue and guarantee. However, under the established layout, how to identify and integrate all kinds of emergency resources in a quick and efficient way and make an emergency resources dispatching plan come into being is always a difficult problem in face of uncertain emergency.

As is known to all, emergency management system is an open and complicated huge system. This system has characteristics like multi-agent, multi-factor, multi-scale, variability and so on, and emergency decision-making under complex conditions, especially emergency resource scheduling decision, is one of the five key problems in emergency management basic research. Based on the dynamic of emergency resources, this paper expounds the identification and integration of emergency resources, and gives a multi-stage dispatching idea of emergency resources based on two-stage emergency rescue process and continuous consumption dynamic structure.

\section{Identification of Dynamic Structure Emergency Resources}

Emergency resources, in a broad sense, all the resources that are required to be used in the event of an emergency response can be called emergency resources, that is, after the public emergencies, in order to ensure the smooth progress of emergency treatment and to maintain people's normal production and life of all the resources needed. Whether these resources are naturally generated or artificially created and whether it is existing or potential, including human, material, financial, information, etc., can be attributed to the scope of emergency resources. Obviously, to improve the comprehensive capacity of emergency resources, reserve a full range, the number of adequate emergency resources is effective to deal with the basis of a variety of public emergencies, is of great significance. Because emergency rescue is usually characterized by multi-agent, multi-factor, multi-scale, variability and so on, and the demand for emergency resources has the characteristics of sudden, uncertain, timeliness, stage and mandatory, it is difficult to carry out accurate prediction. Therefore, people need to change the contingency resources according to the change of the scene situation and the emergency process. An effective way to solve this problem is to change the concept and method of emergency resource integration. Relying on modern 
information fusion technology and modern scientific management methods makes the main body of emergency resources diversification, diversification of forms, content information, to improve the emergency response to emergency response to the ability of emergency management. Because of the difference of time, space, quantity, quality and other attributes of emergency resources, it will lead to the dynamic characteristics of dynamic multidimensional structure. Therefore, it is necessary to construct a dynamic structure emergency resource identification system based on information fusion technology. This is the basis for emergency rescue and resource integration, scheduling. Dynamic structure emergency resource identification system can be mainly from the source of emergency resources identification, demand identification, classification and other aspects of classification.

\subsection{Source Identification}

Emergency resources according to their status can be divided into two categories of current resources and potential resources. Current resources include existing resource reserves, immediate production outputs under known production capacity, predictable immediate financing, etc. Potential resources include a variety of potential resource supplies, such as direct expropriation, emergency expansion, organizational donation, spontaneous provision of resources, etc. Due to different sources can provide the specific resources of different varieties, different numbers, different times, different transport capacity, so the need to rescue the emergency resources on the basis of the identification of effective resources. The purpose of emergency resource integration is to: First, the current emergency resources for the salvage point are effectively integrated according to the emergency needs, so that they are more effectively matched with the resource requirements in quantity, quality and time. Second, for the potential emergency resources can be effectively to confirm and develop a follow-up emergency resource replenishment plan.

In the modern information fusion technology conditions, it can be from the time, space, quantity and other aspects of the emergency resources for character recognition analysis: (1) Time dimension. Mainly refers to the emergency rescue in the emergency resources from mobilization, to raise the time to put into the emergency response process characteristics. It mainly includes the characteristics of resource generation, that is, a rescue point (resource reserve point) of a resource from scratch time. The growth characteristics of a resource, that is, the number of changes in a resource at a given point. Resources can save the state, that is, a rescue point of a resource can be put into the emergency rescue time. (2) Spatial dimension. It mainly points out the characteristics of the geographical distribution of the salvage point, that is, the emergency resource scheduling of a rescue point when the emergency rescue is carried out. (3) The number of dimensions. This includes the total reserves of the current resources, the number of potential resources, and the maximum capacity of the resources based on the maximum transport capacity. It should be noted that the quantitative factors are in the time series on the change, so the number of factors should be combined with time factors to consider.

\subsection{Requirements Identification}

Emergency resource requirements are the minimum resource requirements for effective response to emergencies. The so-called effective, both refers to better benefits and the lower loss, but also refers to the efficient use of emergency resources. Therefore, the emergency resource demand is $y$, the minimum number of demand combinations under the conditions of coping with various types of emergency resources successfull. As the demand for emergency resources has the characteristics of suddenness, uncertainty, timeliness, stage and coercion, the demand combination should change with the development of emergencies and the implementation of emergency rescue.

Emergency resource requirements can be described by quantity, quality, structure and other aspects of the property. (1) Quantity requirements. The size of the emergency requirements is usually related to the size and intensity of emergencies and the occurrence of emergencies. In general, the higher the level of emergencies, the greater the impact, the greater the population density around the incident, the greater the economic and social losses, the greater the number of emergency resource demand. (2) Quality requirements. A certain amount of resource demand is always based on a certain quality assurance requirements. The quality requirements of resources include the timeliness, reliability and cost requirements. The nature of public emergencies, the possible harm, the size of the occurrence and the way it copures will affect the quality of resources needs. (3) Structural requirements. The so-called resource structure needs mainly refers to the emergency rescue and disposal process of various types of resource requirements between the structure of the relationship between the proportion. The types and levels of emergencies usually determine the structural requirements of resources. Different types and levels of emergencies have different combinations of emergency resource requirements.

\subsection{Graded Identification}

Based on the study of the source and demand identification of emergency resources, how to classify all kinds of emergency resources is of great significance to the effective integration and full use of emergency resources. Common emergency resource classification is mainly from the use of resources, urgency, use, demand incentives and other aspects of the division, such as "emergency materials classification and product catalog" is the National Development and Reform Commission announced in accordance with the use of classification of an emergency classification of materials, is currently China's most comprehensive and most authoritative one. On the basis of the classification of emergency resources, combined with the specific situation of emergency resource demand and the characteristics of resources itself, it is very 
important to classify and manage all kinds of emergency resources. In fact, the classification of emergency resources should be based on the characteristics of resources and the types of emergencies, the level of the demand for resources to dynamically determine the characteristics.

Commonly used classification methods are fuzzy clustering analysis, fuzzy comprehensive evaluation method, neural network classification method. These methods are still less applied in the research of emergency resource classification, mainly because the emergencies are non-routine and lack the comprehensive index system of scientific evaluation of emergency resource demand level, and the data on the evaluation index are difficult to collect or because of the data The source and the nature of the reasons difficult to effectively deal with. A feasible approach to the classification of emergency resources is based on the identification of emergency resource sources and needs, from the timeliness of resources, the gap of resources, the availability of resources, the reliability of resources and other indicators to evaluate, based on dynamic Information fusion, the use of evidence reasoning, fuzzy reasoning logic and related management decision-making methods to the emergency resource classification decision-making, in order to achieve the emergency resources of the dynamic classification management and resource security.

\section{Integration of Dynamic Structures Emergency Resources}

In the emergency rescue, the effectiveness of resource scheduling and economy is the core issue of emergency decision-making. On the basis of emergency resource identification, the next question to consider is how to ensure that the resources can be effectively used in emergency rescue. In fact, emergency rescue in order to ensure that the emergency response is not interrupted, it is necessary to ensure the continuity of emergency resources consumption. According to the demand characteristics of the emergency point in the emergency event and the supply status information of the rescue resource, the instantaneous consumption rate of the emergency resources (the amount of consumption per unit time) can be obtained, which stipulates the next emergency resources Must arrive at the time. In addition, in order to effectively carry out emergency resource scheduling, you need to rescue the emergency distribution of resources for dynamic integration, where also need to consider the consumption of resources, scheduling time and speed.

In fact, when we mastered the consumption rate of resources, the potential resources of the generation characteristics, but also can effectively reduce the resource allocation of the rescue points; can also be based on the current resources to determine the amount of resources to save the rescue plan The This is actually a demand-based process management and rate management ideas that focus on the needs and how to ultimately meet the needs of the process, by optimizing the process to replace the number of speed, to improve the efficiency of emergency rescue and benefit.

Consider the two phase of the emergency rescue process. The first stage, when the incident occurred, according to the limited information corresponding to the sharp point, according to the decision rule for minimum response time and maximum carrying amount of resources, select the number of depots to emergency transportation related emergency resources, and will be the first batch of emergency resources to reach the emergency and just be consumed and this time is denoted as to time (i.e., the second batch of resources should arrive at the moment). The second stage, considering the consumption rate of the various emergency emergency resources and the maximum carrying capacity constraints. As the first batch of resources consumed just moments is t0 time, we can determine the second batch of resource was consumed time is denoted as $\mathrm{t} 1$. Remembe that each following batch of emergency resources is consumed exactly and this moment is $\mathrm{t} 2, \ldots, \mathrm{TN}$. There is the need to ensure that at any moment has arrived at the amount of resources to meet the emergency rescue in the consumption of resources, avoid suspension of emergency activities caused by insufficient supply of resources, that is to say, ensure that every two resource scheduling of arrival time interval delta TI 'is no more than one scheduling resource consumption time delta ti=ti-ti- 1 . Obviously, between the second stages of each batch of emergency emergency resources just consumed from the time point of the delta ti= delta TI 'difference, can guarantee the maximum utilization of emergency resources. In particular, when delta ti is constant delta $\mathrm{T}$, can guarantee the continuity and stability of emergency disposal. In order to ensure this, it is necessary to use the dynamic integration of each batch of emergency resources and the dynamic integration of the rescue point to balance the actual time difference of each resource consumption. The effective integration of emergency resources are mainly from the following aspects.

\subsection{The Dynamic Integration of the Rescue Point}

The dynamic integration of the rescue points is to determine the set of dispatch points for each dispatch. Based on the two-stage emergency rescue process, the first stage involves only one resource scheduling, while the second stage involves multiple resource scheduling.

In the first stage, the distance between the emergency resource status information, the transport capacity and the rescue point and the emergency point are taken into account, and the minimum emergency response time and the maximum carrying capacity are taken as the goal. By establishing a certain mathematical programming model, And through the design of the corresponding algorithm to select the appropriate rescue point program.

The second stage of the rescue point of choice, mainly based on the first stage of the rescue point, the rescue point of resources reserves (remaining) amount, the emergency point of the consumption rate of various resources, potential rescue points of resources (remaining) quantity, the rescue point and the potential rescue point of the transport capacity, the rescue point and the potential rescue point and the emergency point 
distance and other factors to decide, mainly the following research: 1. emergency resource consumption rate of dynamic integration. Mainly to consider the emergency point of the dynamic demand for various resources, consumption rate, the overall grasp of the emergency point of consumption of resources. 2. to determine the set of rescue points. According to the premise of the first stage of the rescue point, under the premise of ensuring $\Delta \mathrm{ti} \leq \Delta \mathrm{ti}$ ', the first is based on the demand of the emergency point with the dynamic change, the consumption rate of the emergency point and the resource surplus of the rescue point The situation to determine whether the rescue resources are sufficient If sufficient, then the aforementioned rescue point will a candidate to; if not enough, then consider the distance between the rescue and emergency, the rescue point of the ability to carry out the potential to save the point and the rescue point and the distance The potential to save the point of carrying capacity to determine the potential rescue points and the plan of rescue points and whether to join the next batch of alternative rescue points set or not. In general, from the time (scheduling time), the number (emergency resources and structure) and cost (the number of rescue points and transport capacity) these three goals are mainly to dynamically determine the rescue and potential rescue points.

\subsection{To Save the Point of Dynamic Integration of Resources}

From the point of view of the dynamic change of the number of emergency resources to balance the actual time of the emergency consumption of resource inconsistencies, which can guarantee the consistency of time $\Delta t$. To this end, to determine the rescue points each time the amount of resources to save the resources of the integration of the core part of the research content, the main need to consider: 1 . save the point reserve (surplus) resources. Considering the structural characteristics of the reserve resources (residual) resources and emergency resource requirements, the resources of each rescue point are integrated from the three aspects of the category, quantity and location, and the resource status of the reserve (reserve) is grasped as a whole. 2. save the rescue resources. Based on the comparison between emergency resource demand and reserve (surplus) resources, the number of rescue resources is determined according to the factors such as carrying capacity and transportation time.

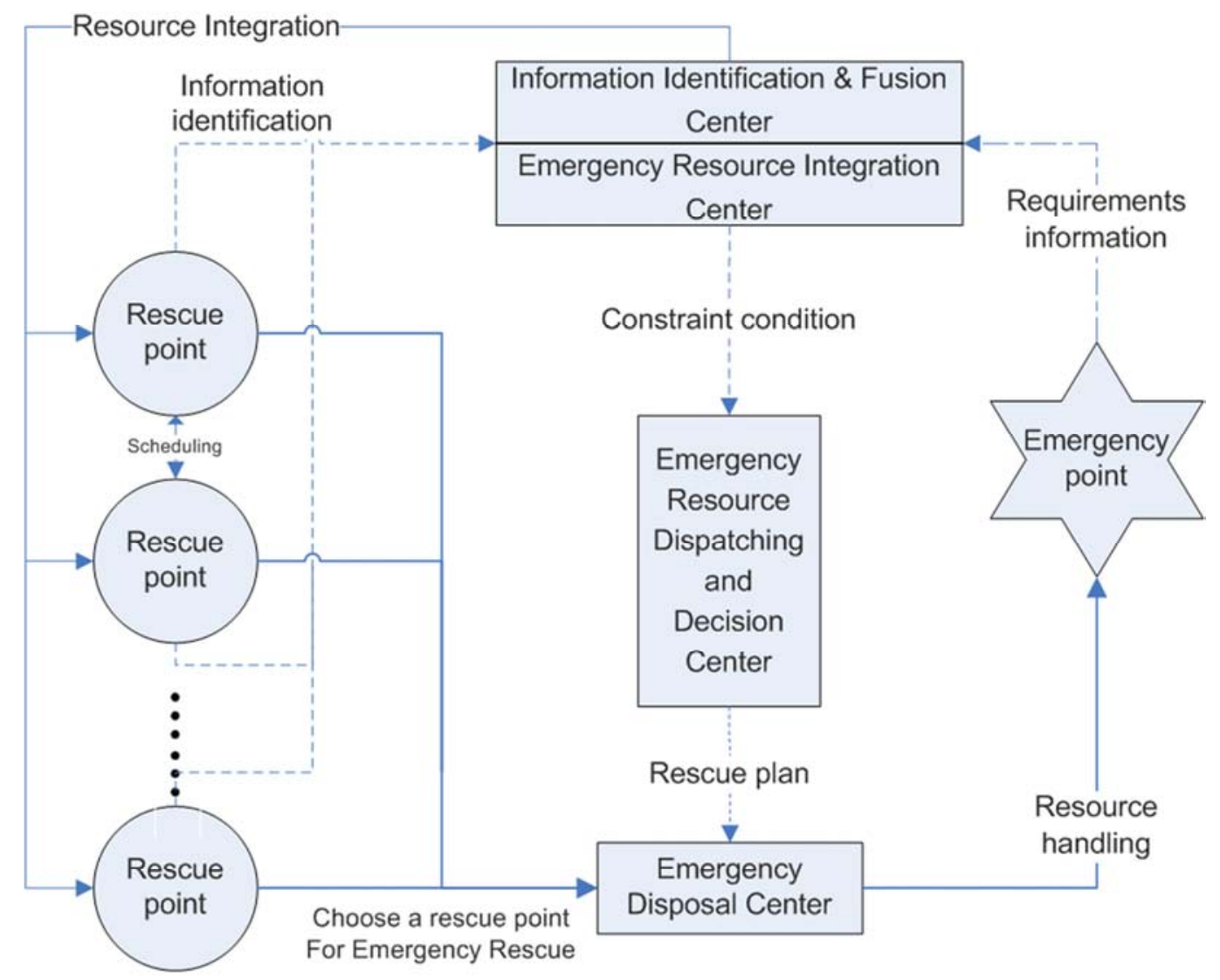

Figure 1. Dynamic resource emergency resource scheduling process based on information fusion and continuous consumption

\section{Conclusion}

The above information based on information fusion and continuous consumption of dynamic structural emergency resource scheduling process is shown in Figure 1. The characteristics of this idea are: each batch of resource scheduling from the resource needs of the structure to ensure that the consumption of resources is the same time, or that the rescue point to reach the emergency point of time is the same. In practice, if the choice of a number of rescue points out of the program, due to the rescue point to reach the emergency 
point of the distance, the path is inconsistent, the rescue point to reach the point of time is also inconsistent, the problem is actually Disposable scheduling model to ensure the continuity of resource consumption. The problem can be solved by the refinement of the scheduling decision model or the optimal design of the emergency workflow. In the emergency rescue practice, the emergency disposal needs to take the random destructive (transfer efficiency), capacity limitation and emergency disposal capacity of the resource scheduling path into account. In addition, the flexibility, reliability and application of the emergency system Sex is also an important issue to consider.

\section{Acknowledgement}

Supported by National Natural Science Foundation of China (71101076);

Supported by the MOE Layout Foundation of Humanities and Social Sciences (10YJA630147);

Supported by Natural Science Foundation of Nantong University (10ZY002).

\section{References}

[1] Fan Weicheng. Advisement and Suggestion to Scientific Problems of Emergency Management for Public Incidents [J]. Bulletin of National Natural Science Foundation of China, 2007, (2): 71-76.

[2] Shi Bo, Liang Jingguo, Liu Tuo. Study on the Principle of Resource Allocation in Emergency Management of Public Crisis [J]. Modern Management Science, 2008, (6): 88-89.
[3] Liu Chunlin, He Jianmin, Sheng Zhaohan. Fuzzy Programming Method for Emergency System Scheduling Problem [J]. Journal of Systems Engineering, 1999, 14 (4): 351-355.

[4] Wang Yu, He Jianmin. Research on Multi-resource Saving Scheme in Emergency System [J]. Journal of Southeast University (English Edition), 2002, 32 (3), 510-513.

[5] $\mathrm{Hu}$ Feihu, etc. Research on Multi-objective Emergency Scheduling Problem with Multi-resource Combination [J]. Microcomputer Information (Management-Control Integration), 2009, 25 (7-3): 9-10.

[6] Fiedrich F, Gehbauer F, Rickers U. Optimized Resource Allocation for Emergency Response after Earthquake Disasters [J]. Safety Science, 2000, 35 (1): 41-57.

[7] Yu Yingying, etc. The Model and Algorithm of Resource Layout Evaluation and Adjustment in Emergency Management [J]. Systems Engineering, 2008, 26 (1): 75-81.

[8] Liu Chunlin, He Jianmin, Sheng Zhaohan. The Selection of the Optimal Scheme for the Emergency System [J]. Journal of Industrial Engineering and Engineering Management, 2000, 14 (1): $13-15$.

[9] Shetty S R. An event driven single game solution for resource allocation in a multi-crisis environment [D]. Tampa; Univ South Florida, 2004.

[10] Zhang Zhiqing, Xi Bao, Yang Zhonghua, Wang Xinxin. Supply Chain Collaborative Demand Forecasting Based on Information Fusion [J]. Science and Technology Progress and Policy, 2008, 25 (12): 174-177.

[11] Wu Shihui, Yang Jianjun. Research on Dispatching Problem of Emergency System Resource for One - time Consumption [J]. Logistics Sci-tech, 2009 (7): 47-49. 